\title{
ALGEBRAS OF INTEGRABLE FUNCTIONS. II
}

\author{
KENNETH O. LELAND ${ }^{1}$
}

1. Introduction. Morera's theorem in complex function theory raises the possibility that this theory can be based on integration rather than differentiation. Heffter [1], Macintyre and Wilbur [9] and this author [7] have given such a development. In this paper a theory of integrable functions will be developed in a more general context of operator valued functions wherein the functions no longer need be analytic.

Let $K$ denote the complex plane. For $a \in K$, set $A_{a}(z)=a z$ for all $z \in K$. Then $A_{a}$ is a bounded linear transformation of $K$, thought of as a real Euclidean space $E_{2}$ into itself. Set $T_{2}{ }^{\prime}=\left\{A_{a} ; a \in K\right\}$. Let $f$ be a continuous function on an open set $S$ in $E_{2}$ into the space $B_{2}$ of bounded linear transformations of $E_{2}$ into itself, and let $P$ be a path (rectifiable arc) in $S$ with endpoints $\alpha$ and $\beta$. Then for any subdivision $\alpha=z_{1}<\cdots<z_{n+1}=\beta$ of $P$, a Riemann sum, the vector $R=$ $\sum_{i=1}^{n} f_{z_{i}}\left(z_{i+1}-z_{i}\right)$ can be formed. If range $f$ lies in $T_{2}{ }^{\prime}$, then, for $z \in \mathrm{S}, f_{z}=f(z)=A_{\phi(z)}$ for some $\phi(z) \in K$, and we may write $R=$ $\sum_{i=1}^{n} \phi\left(z_{i}\right)\left(z_{i+1}-z_{i}\right)$. Taking the limit as the norm of the subdivision defining $R$ approaches zero, we obtain the vector $\theta=\int_{\alpha_{p}}^{\beta} f(z) d z$ $=\int_{\alpha_{p}}^{\beta} f_{z}(d z)$. If range $f \subseteq T_{2}{ }^{\prime}$, we can interpret $\theta$ as the complex number $\int_{\alpha_{p}}^{\beta} \phi(z) d z$.

$f$ is said to be integrable if for all closed paths (rectifiable simple closed curves) $C \subseteq S$, we have $\int_{C} f(z) d z=\int_{C} f_{z}(d z)=0$. If range $f \subseteq T_{2}{ }^{\prime}$, then $\int_{C} \phi(z) d z=0$ for all closed paths $C \subseteq S$, and by Morera's theorem $\phi$ is analytic; consequently, $f$ is itself Fréchet differentiable, where $f_{z}{ }^{\prime}$ is a linear transformation of $E_{2}$ into $B_{2}$ for $z \in S$.

The general case studied in this paper is obtained by replacing $E_{2}$ by an arbitrary real Euclidean space $E$ of dimension $p, p>1$. Let $T$ be a commutative subalgebra of the Banach algebra $B$ of bounded linear transformations of $E$ into $E$ and let $F$ be the family of continuous integrable functions on open subsets of $E$ into $T$.

Let $E^{\prime}$ be a finite-dimensional commutative Banach algebra. with identity over the reals and for $a \in E^{\prime}$, set $A_{a}(t)=a t$ for $t \in E^{\prime}$. Set $T^{\prime}$ $=\left\{A_{a} ; a \in E^{\prime}\right\}$.

Received by the editors August 18, 1970 and, in revised form, November 12, 1970.

AMS 1970 subject classifications. Primary 30A96, 30A98; Secondary 30A90.

${ }^{1}$ This research supported by National Science Foundation Grant GP-6311.

Copyright $\odot 1972$ Rocky Mountain Mathematics Consortium 
Let $f \in F, S=\operatorname{domain} f$ simply connected. Let $z_{0} \in S$ and for $z \in S$, set $g(z)=\int_{z_{0}}^{z} f(z) d z$. Then $g$ maps $S$ into $E$, and for $z \in S$, the Fréchet derivative $g_{z}{ }^{\prime}$ of $g$ at $z$ is the operator $f(z)=f_{z}$ of $T$.

It shall be shown in $\$ 2$, that the family of continuous integrable functions on a simply connected open set $S$ in $E$ into $T$ forms an algebra. The method of proof is reminiscent of the proof of the Cauchy-Goursat theorem. For the case that $E=E_{2} \cong K$, one may refer to [7].

In $\S 3$, the relationship between integrability and differentiability is discussed. Examples of integrable but nondifferentiable functions are given.

In $\S 4, T$ is required to be symmetric, that is, for $x \in T$, the adjoint $x^{*}$ of $x$ lies in $T$. In this case questions concerning the analyticity or nonanalyticity of elements of $F$ can be readily answered since in this case an element of $F$ can be expressed locally as a direct sum of functions which can be interpreted as analytic functions from $K$ into $K$ or as continuous functions from $R$ into $R$. This analysis is based on the fact that $E$ can be expressed as the direct sum of irreducible subspaces invariant under $T$.

In $\$ 5$, it is shown that provided that $T$ is presumed semisimple, a necessary and sufficient condition for analyticity of the elements of $F$ is that no element of $T$ have rank one (i.e. range $A=A(E)$ is not one dimensional for all $A \in T)$. The possibility that the presumption of semisimplicity may be dispensed with is examined.

Employed in $\$ 5$ is a definition of analyticity in a real variable context suitable for the purposes of this paper due to the author [2], [5], [6] .

In [8] it shall be shown that integrable functions, although not necessarily analytic, satisfy some form of the maximum modulus theorem.

The development of this paper is in no way affected if the only paths of integration permitted are those formed from straight line segments.

Let $\omega$ denote the positive integers. If $Z$ is a Banach space, $\delta>0$, $x \in Z$, set $U_{x}(\delta)=\{t \in Z ;\|t-x\|<\delta\}, U(\delta)=U_{0}(\delta)$ and $U=U_{0}(1)$, and set $V_{x}(\delta)=\{t \in Z ;\|t-x\|=\delta\}, V(\delta)=V_{0}(\delta)$, and $V=V_{0}(1)$. If $f$ is a function with domain $S$ and $H \subseteq E$, then the restriction $f \mid H$ of $f$ to $H$ is the function $g$ with domain $H \cap S$ such that $g(x)=f(x)$ for all $x \in H \cap S$. For $H$ a subspace of $E$ and $G$ a family of functions defined on subsets of $E, G \mid H$ denotes the family $\{g \mid H ; g \in G\}$.

A subspace $H$ of $E$ is said to be invariant if $A(x) \in H$ for all $A \in T$, $x \in H$.

2. The algebras. The principal result of this section, Theorem 2.1, is the proof that the product of two elements of $F$ with common simply 
connected domains also lies in $F$. Our basic tool is the "smoothing" lemma, Lemma 2.1, which allows us to approximate elements of $F$ by "smoothed" elements of $F$ satisfying a Lipschitz type condition, enabling us to use arguments reminiscent of the proof of the CauchyGoursat theorem to achieve our conclusion.

Trivially $\boldsymbol{F}$ is closed under the operations of addition (defined on the intersection of the domains of the functions being added), multiplication by scalars, and translations of the form $x \rightarrow x-x_{0}, x, x_{0} \in E$. It may be readily shown that if $f_{1}, f_{2}, \cdots$ is a sequence of elements of $F$ with common domain $S$ which converges uniformly on $S$ to a limit function $f$, then $f$ also lies in $F$.

LeMma 2.1. Let $f \in F$ and let $H$ be a compact subset of domain fwith interior $S$. Then there exists a sequence $g_{1}, g_{2}, \cdots v j$ elements of $F$ with common domain S such that:

(1) The sequence $g_{1}, g_{2}, \cdots$ converges uniformly on $S$ to $f \mid S$.

(2) For $i \in \omega$, there exists $N_{i}>0$ such that for $x, y \in S$,

$$
\left\|g_{i}(y)-g_{i}(x)\right\| \leqq N_{i}\|y-x\|
$$

Our proof is based upon the discussion of "smoothing" operators as introduced by the author in [5] .

Proof. Let $e_{1}, \cdots, e_{p}$ be an orthonormal basis of $E$ and let $Q$ be the cube $\left\{-\frac{1}{2}<\left(x, e_{i}\right) \leqq \frac{1}{2} ; i=1, \cdots, p\right\}$. For $x \in E, s>0$ such that $x+s \bar{Q}=\{x+s y ; y \in \bar{Q}\} \subseteq$ domain $f$, set

$$
f_{s}(x)=s^{-p} \int_{\dot{x}+s Q} f(t) d m(t),
$$

where $m$ is Lebesgue measure on $E$. Let $\alpha>0$,

$$
\alpha<\inf \{\|x-y\| ; x \in H, y \in E-\operatorname{domain} f\}
$$

and set $H_{0}=H+a \bar{Q}$, where $a=\alpha / \vee p$. Then $H_{0}$ is compact and $H \subseteq H_{0} \subseteq$ domain $f$. Let $0<s \leqq a$. Then $H \subseteq$ domain $f_{s}$.

We now show that $f_{s} \mid S$ is the uniform limit of a sequence of elements of $F$ with common domain $S$ and hence $f_{s} \mid S$ lies in $F$. Let $\epsilon>0$. Then there exits $\delta>0$, such that $x, y \in H_{0}, \quad\|x-y\|<\delta$ implies $\|f(y)-f(x)\| \leqq \epsilon$. Let $0<r<\delta / \sqrt{ } p$ and $t_{1}, \cdots, t_{n} \in s Q, n \in \omega$, such that $\left\{t_{i}+r Q ; i=1, \cdots, n\right\}$ is a partition of $s Q$. Then for $x$ $\in H \subseteq H_{0}, i=1, \cdots, n, t \in x+t_{i}+r Q \subseteq x+s Q \subseteq H+s Q \subseteq H_{0}$, we have $\left\|t-\left(x+t_{i}\right)\right\| \leqq r \sqrt{ } p<\delta$ and hence $\left\|f(t)-f\left(x+t_{i}\right)\right\| \leqq \epsilon$. Thus for $x \in H$, 


$$
\begin{aligned}
& \left\|\left[\sum_{1}^{n}(r / s)^{p} f\left(x+t_{i}\right)\right]-f_{s}(x)\right\| \\
& =s^{-p}\left\|\left[\sum_{1}^{n} r^{p} f\left(x+t_{i}\right)\right]-\left[\sum_{1}^{n} \int_{x+t_{i}+r Q} f(t) d m(t)\right]\right\| \\
& =s^{-p}\left\|\sum_{1}^{n} \int_{x+t_{i}+r Q}\left[f\left(x+t_{i}\right)-f(t)\right] d m(t)\right\| \\
& \leqq s^{-p} \sum_{1}^{n} \int_{x+t_{i}+r Q}\left\|f\left(x+t_{i}\right)-f(t)\right\| d m(t) \\
& \leqq s^{-p} \sum_{1}^{n} r^{p} \epsilon=s^{-p}\left(n r^{p}\right) \epsilon=s^{-p}\left(s^{p}\right) \epsilon=\epsilon
\end{aligned}
$$

where $n r^{p}$ is clearly the volume of $s Q$. Thus $f_{s} \mid H$ is the uniform limit on $H$ of elements of $F$ of the form $g(x) \equiv \sum_{1}^{n}(r / s)^{p} f\left(t_{i}+x\right)$, and thus $f_{s} \mid S$ lies in $F$.

Now for $x \in H, s<\min \{a, \delta / \vee p\}, s>0$,

$$
\left\|f(x)-f_{s}(x)\right\|=s^{-p}\left\|\int_{x+s Q}[f(x)-f(t)] d m(t)\right\| \leqq s^{-p}\left(\epsilon s^{p}\right)=\epsilon .
$$

Let $0<s \leqq a$. We now verify (2) for $f_{s} \mid S$. Now there exists $N_{s}>0$, such that $m[(x+s Q) \dot{+}(y+s Q)] \leqq N_{s}\|y-x\|, \quad$ for $x, y \in E$, where $A+B=(A-B) \cup(B-A)$ for arbitrary sets $A, B$. Thus for $x, y \in H$,

$$
\begin{aligned}
\left\|f_{s}(y)-f_{s}(x)\right\| & =s^{-p}\left\|\int_{y+s Q} f(t) d m(t)-\int_{x+s Q} f(t) d m(t)\right\| \\
& \leqq s^{-p} \int_{(x+s Q) \dot{+}(y+s Q)}\|f(t)\| d m(t) \\
& \leqq s^{-p} m[(x+s Q) \dot{+}(y+s Q)] M \\
& \leqq s^{-p}\left[N_{s}\|y-x\|\right] M \leqq N_{s}^{\prime}\|y-x\|,
\end{aligned}
$$

where $M=\sup \left\{\|f(t)\| ; t \in H_{0}\right\}$, and $N_{s}{ }^{\prime}=N_{s} M s^{-p}$.

For $n \in \omega, x \in S$, set $g_{n}(x)=f_{a / n}(x)$. Then $g_{1}, g_{2}, \cdots$ is the desired sequence of functions.

Theorem 2.1. Let $S$ be a simply connected open set in $E$ and $f, g$ elements of $F$ with domain $S$. Then $f g \in F$.

Proof. It suffices to show that $\int_{\Delta} f(z) g(z) d z=0$ for a triangle $\Delta$ such that $\Delta$ and its interior $J$ lie in $S$. Now there exists an open set 
$D \subseteq S$, with $\bar{D}$ compact, such that $\bar{J} \subseteq D$ and $\bar{D} \subseteq$ S. Let $f_{1}, f_{2}, \cdots$ and $g_{1}, g_{2}, \cdots$ be the sequences of functions given by Lemma 2.1 for $f$ and $g$ and $H=\bar{D}$. If we show for all $n \in \omega$, that $\int_{\Delta} f_{n}(z) g_{n}(z) d z$ $=0$, then

$$
\int_{\Delta} f(z) g(z) d z=\lim _{n \rightarrow \infty} \int_{\Delta} f_{n}(z) g_{n}(z) d z=\lim _{n \rightarrow \infty} 0=0 .
$$

Fix $n \in \omega$. Then from Lemma 2.1, there exist $N_{n}, M_{n}>0$, such that for $x, y \in$ interior $\bar{D} \supseteq D \supseteq \bar{J}$,

$$
\left\|f_{n}(y)-f_{n}(x)\right\| \leqq N_{n}\|y-x\| \text { and }\left\|g_{n}(y)-g_{n}(x)\right\| \leqq M_{n}\|y-x\| \text {. }
$$

Let $k \in \omega$, and divide $\Delta$ into $4^{k}$ congruent triangles of perimeter $s / 2^{k}, \Delta_{1}, \cdots, \Delta_{4^{k}}$, where $s$ is the perimeter of $\Delta$, and let $z_{i} \in \Delta_{i}$, for $i=1, \cdots, 4^{k}$. Now for $i=1, \cdots, 4^{k}, f_{n}\left(z_{i}\right) g_{n}, f_{n} g_{n}\left(z_{i}\right)=$ $g_{n}\left(z_{i}\right) f_{n}$, and $f_{n}\left(z_{i}\right) g_{n}\left(z_{i}\right)$ are integrable and hence

$$
\int_{\Delta_{i}} f_{n}(z) g_{n}(z) d z=\int_{\Delta_{i}}\left[f_{n}(z)-f_{n}\left(z_{i}\right)\right]\left[g_{n}(z)-g_{n}\left(z_{i}\right)\right] d z,
$$

and thus

$$
\begin{aligned}
& \left\|\int_{\Delta_{i}} f_{n}(z) g_{n}(z) d z\right\| \leqq \int_{\Delta_{i}}\left\|f_{n}(z)-f_{n}\left(z_{i}\right)\right\| \cdot\left\|g_{n}(z)-g_{n}\left(z_{i}\right)\right\| d s \\
& \quad \leqq \int_{\Delta_{i}} N_{n}\left\|z-z_{i}\right\| \cdot M_{n}\left\|z-z_{i}\right\| d s \leqq \int_{\Delta_{i}} N_{n}\left(s / 2^{k}\right) \cdot M_{n}\left(s / 2^{k}\right) d s \\
& \quad \leqq\left[N_{n} M_{n} s^{2} / 4^{k}\right]\left(s / 2^{k}\right)=N_{n} M_{n} s^{3} / 8^{k} .
\end{aligned}
$$

Thus

$$
\begin{aligned}
\left\|\int_{\Delta} f_{n}(z) g_{n}(z) d z\right\| & =\left\|\sum_{i=1}^{4^{k}} \int_{\Delta_{i}} f_{n}(z) g_{n}(z) d z\right\| \\
& \leqq \sum_{i=1}^{4^{k}}\left\|\int_{\Delta_{i}} f_{n}(z) g_{n}(z) d z\right\| \\
& \leqq 4^{k}\left[N_{n} M_{n} s^{3} / 8^{k}\right]=N_{n} M_{n} s^{3} / 2^{k} .
\end{aligned}
$$

Letting $k \rightarrow \infty$, we obtain $\int_{\Delta} f_{n}(z) g_{n}(z) d z=0$.

REMark. In his argument for the complex function case Heffter [1] requires that one of the functions $f, g$ be differentiable. Macintyre and Wilbur [9] weaken this, requiring only that one of the functions satisfy a Lipschitz condition. The need for such additional assumptions has been obviated in our argument by the use of the "smoothing" lemma, Lemma 2.1. Commutativity comes into play only once, appearing in the argument that functions of the form $f(z) c$, where $f$ is integrable and $c \in T$, are also of the form $c f(z)$ and hence integrable. 
Corollary 2.1. Let $f$ be an element of $F$ with simply connected domain $S$ such that $g(z)=f(z)^{-1}$ exists for all $z \in S$. Then $g \in S$.

Proof. Let $\Delta, J, D, f_{1}, f_{2}, \cdots$ be as given in the proof of Theorem 2.1. Now $f(\bar{J})$ is a compact subset of the open set $Z$ of invertible elements of $T$. Hence there exists $\delta>0$, such that $x \in f(\bar{J}), z \in T$, $\|z-x\|<\delta$ implies $z \in Z$. Since the sequence $f_{1}, f_{2}, \cdots$ converges uniformly on $\bar{J}$, there must exist $q \in \omega$, such that $f_{n}(x) \in Z$ for $x \in \bar{J}$, $n \in \omega_{q}=\{n \in \omega ; n \geqq q\}$. For $n \in \omega_{q}, x \in \bar{J}$, set $g_{n}(x)=f_{n}(x)^{-1}$. Now for $n \in \omega_{q}$, there exists $N_{n}>0$ such that for $x, y \in \bar{J}, \| f_{n}(y)-$ $f_{n}(x)\left\|\leqq N_{n}\right\| y-x \|$, and hence

$$
\begin{aligned}
\left\|g_{n}(y)-g_{n}(x)\right\| & =\left\|g_{n}(y) g_{n}(x)\left[f_{n}(x)-f_{n}(y)\right]\right\| \\
& \leqq\left(N_{n}{ }^{\prime}\right)^{2}\left[N_{n}\|y-x\|\right]=N_{n}{ }^{\prime \prime}\|y-x\|,
\end{aligned}
$$

where $N_{n}{ }^{\prime}=\sup \{\|g(t)\| ; t \in \bar{J}\}$ and $N_{n}{ }^{\prime \prime}=\left(N_{n}{ }^{\prime}\right)^{2} N_{n}$.

Let $k \in \omega$ and divide $\Delta$ into $4^{k}$ congruent triangles of perimeter $s / 2^{k}, \Delta_{1}, \cdots, \Delta_{4^{k}}$, where $s$ is the perimeter of $\Delta$, and let $z_{i} \in \Delta_{i}$ for $i=1, \cdots, 4^{k}$. Now for $i=1, \cdots, 4^{k}, g_{n}\left(z_{i}\right) f_{n}$ is integrable and hence

$$
\begin{aligned}
& -g_{n}\left(z_{i}\right) \int_{\Delta_{i}}\left[f_{n}(z)-f_{n}\left(z_{i}\right)\right]\left[g_{n}(z)-g_{n}\left(z_{i}\right)\right] d z \\
& \quad=-g_{n}\left(z_{i}\right) \int_{\Delta_{i}}-f_{n}\left(z_{i}\right) g_{n}(z) d z=\int_{\Delta_{i}} g_{n}(z) d z .
\end{aligned}
$$

Then $\quad\left\|\int_{\Delta_{i}} g_{n}(z) d z\right\| \leqq N_{n}{ }^{\prime}\left[N_{n} N_{n}{ }^{\prime \prime}\right] s^{3} / 8^{k} \quad$ and $\quad\left\|\int_{\Delta} g_{n}(z) d z\right\| \leqq$ $N_{n} N_{n}{ }^{\prime} N_{n}{ }^{\prime \prime} s^{3} / 2^{k}$. Letting $k \rightarrow \infty$ and then letting $n \rightarrow \infty$, the theorem follows.

Let $A$ be a path in $E^{\prime}$ with endpoints $\alpha$ and $\beta$ and let $f$ and $g$ be continuous functions on $A$ and $A \times A$ respectively into $E^{\prime}$. Then clearly $\theta=\int_{\alpha_{A}}^{\beta} f(z) d z$ is defined, where $A_{\theta}=\int_{\alpha_{A}}^{\beta} A_{f(z)}(d z)$. Let $P: \alpha=x_{0}<\cdots<x_{n+1}=\beta, n \in \omega$, be a subdivision of $A$ and set

$$
\begin{aligned}
R & =\sum_{i=0}^{n} \sum_{j=0}^{n} g\left(x_{i}, x_{j}\right)\left(x_{i+1}-x_{i}\right)\left(x_{j+1}-x_{j}\right) \\
& =\sum_{i=0}^{n}\left[\sum_{j=0}^{n} g\left(x_{i}, x_{j}\right)\left(x_{j+1}-x_{j}\right)\right]\left(x_{i+1}-x_{i}\right) .
\end{aligned}
$$

Then clearly as the norm of $P$ converges to zero, $R$ converges to a limit $\mu=\int_{\alpha}^{\beta} \int_{\alpha_{A \times A}}^{\beta} g(x, y) d x d y$. Clearly $\mu=\int_{\alpha}^{\beta}\left[\int_{\alpha_{A}}^{\beta} g(x, y) d x\right] d y$ $=\int_{\alpha_{A}}^{\beta}\left[\int_{\alpha_{A}}^{\beta} g(x, y) d y\right] d x$.

A continuous function $f$ on an open set $S \subseteq E^{\prime}$ into $E^{\prime}$ is said to be integrable if $\int_{C} f(z) d z=0$ for all paths $C \subseteq S$. 
Theorem 2.2. Let $S$ be a simply connected open set in $E^{\prime}, 0 \in S$, $f$ a continuous integrable function on $S$ into $E^{\prime}$, and set $P(z)=$ $\int_{0}^{z} f(x) d x$ for $z \in S$. Then $P$ is an integrable function on $S$ into $E^{\prime}$.

Proof. Our argument is based on that of [9]. Let $z \in S$ and let $A$ be a path in $S$ with endpoints 0 and $z$. Then

$$
\begin{aligned}
\int_{0}^{z} P(x) d x & =\int_{0_{A}}^{z}\left[\int_{0_{A}}^{x} f(t) d t\right] d x=\int_{0_{A}}^{z}\left[\int_{t_{A}}^{z} f(t) d x\right] d t \\
& =\int_{0_{A}}^{z} f(t)\left[\int_{t_{A}}^{z} e^{\prime} d x\right] d t=\int_{0_{A}}^{z} f(t)(t-z) d t
\end{aligned}
$$

where $e^{\prime}$ is the identity element of $E^{\prime}$. Set $g(t)=t-z$ for $t \in E^{\prime}$. Then trivially $g$ is integrable, and thus $A_{f}$ and $A_{g}$ are integrable. From Theorem 2.1, $A_{f g}=A_{f} A_{g}$ is integrable and thus $f g$ is integrable and thus the last integral of $(1)$ is dependent of the choice of path $A$ linking 0 and $z$, and consequently $P$ is integrable.

Remark. The requirement that $E$ be finite dimensional may be removed in this section. Suppose $S$ is a simply connected open set in $E$ and $f$, g elements of $F$ with domain $S$, and let $C$ be a closed path in $S$. Then there exists a sequence of closed paths $C_{1}, C_{2}, \cdots$, formed from straight line segments which approximate $C$ such that $\int_{C_{n}} f(z) d z \rightarrow \int_{C} f(z) d z$ as $n \rightarrow \infty$, etc. Now for $n \in \omega$, there exists a finite-dimensional subspace $E_{n}$ of $E$ containing $C_{n}$. Lemma 2.1 readily generalizes to the case of $f / E_{n}$ and $g / E_{n}$, and thus Theorem 2.1 can be reworked to yield $\int_{C_{n}} f(z) g(z) d z=0$. Taking the limit as $n \rightarrow \infty$, we obtain $\int_{C} f(z) g(z) d z=0$. Thus Theorem 2.1 generalizes to the infinite-dimensional case. Corollary 2.1 and Theorem 2.2 similarly generalize.

3. Nonanalytic examples. So far in our discussion no use of the notion of differentiation has been made. Indeed it is quite easy to construct integrable functions which are not differentiable. For $z=(x, y) \in E_{2}=R \oplus R$, set $P(z)=(x, 0)$, and set $T=\{r e+s P$; $r, s \in R\}$. Since $P^{2}=P, T$ is a commutative Banach algebra with identity. Let $h$ be an arbitrary continuous function with domain $R$ into $R$ and set $f(z)=h(x) P$ for $z=(x, y) \in E_{2}$. We show that $f$ is integrable. For $x \in R$, set $u(x)=\int_{0}^{x} h(t) d t$ and for $z=(x, y) \in E_{2}$, set $\boldsymbol{\phi}(z)=[u(x), 0]$. Then for $z=(x, y), \rho=(s, t)$ lying in $E_{2}$, we have $\phi_{z}{ }^{\prime}(\boldsymbol{\rho})=\left[u^{\prime}(x) s, 0\right]=[h(x) s, 0]=h(x) P(\rho)=f_{z}(\boldsymbol{\rho})$, and thus $\phi_{z}{ }^{\prime}=f(z)$. Then clearly for any path $A \subseteq E_{2}$ with endpoints $\alpha$ and $\boldsymbol{\beta}, \int_{\alpha_{A}}^{\beta} f(z) d z=\phi(\beta)-\phi(\alpha)$ and thus for any closed path $C$, $\int_{C} f(z) d z=0$. 
Alternately suppose $C$ is a closed path composed of line segments, none parallel to $\{0\} \times R$. Then $\int_{C} f(z) d z$ can be expressed as $\mu \equiv(1,0) \sum_{0}^{n} \int_{x_{i}}^{x_{i+1}} h(x) d x$, where $x_{0}, x_{1}, \cdots, x_{n+1}=x_{0}, n \in \omega$, are the projections of the endpoints of segments of $C$ onto $R$. Since $x_{n+1}=x_{0}, \mu=0$.

A continuous function $f$ on an open set $S \subseteq E^{\prime}$ is said to be $E^{\prime}$ differentiable if $f$ is (Fréchet) differentiable, and if for all $x \in S$, there exists $c \in E^{\prime}$, such that $f_{x}{ }^{\prime}(t)=c t$ for all $t \in E$. An $E^{\prime}$-differentiable function is integrable. The standard Cauchy-Goursat argument for the complex case [1], [9] involving nesting of triangles readily transfers over to the context of $E^{\prime}$. In this more general context, however, $E^{\prime}$-differentiability does not imply analyticity. Let $E^{\prime}$ be the algebra generated by $e$ and $\mu$, where $\mu^{2}=0$ and $e \mu=\mu e=\mu$. Let $h$ be an arbitrary continuous function with domain $R$ into $R$, and set $u(x)=\int_{0}^{x} h(t) d t$ for $x \in R$. For $z=x e+y \mu \in E^{\prime}$, set $\phi(z)=$ $u(x) \mu$. Then for $z=x e+y \mu, \rho=r e+s \mu \in E^{\prime}, \phi_{z}{ }^{\prime}(\rho)=\left[u^{\prime}(x) r\right] \mu$ $=h(x) r \mu=[h(x) \mu] \rho$, where $h(x) \mu \in E^{\prime}$. Thus $\phi$ is $E^{\prime}$-differentiable and hence integrable; however $\phi^{\prime \prime}$ need not exist, since $h^{\prime}$ need not exist.

If one restricts himself to complex Euclidean spaces $K_{n}, n \in \omega$, (which may be interpreted as real Euclidean spaces $E_{2 n}$ ) and complex homogeneous operators, then the differentiability of the elements of $F$ follows from the fact that the integrals of the elements of $F$ are complex (Fréchet) differentiable and hence infinitely differentiable and analytic. In $\$ 5$ we will show how to insure analyticity by imposing much less drastic conditions on $E$ and $T$ working in a real variable context.

4. The symmetric case. If $E$ can be represented as a direct sum of invariant subspaces, then for $f \in F, f$ can be expressed as a direct sum of integrable functions defined on the subspaces. If $T$ is symmetric, then $E$ can be expressed as the direct sum of mutually orthogonal one- and two-dimensional invariant subspaces, and for $f \in F, f$ can be expressed as the direct sum of functions which can be interpreted as continuous functions on $R$ into $R$ or as complex analytic functions on $K$ into $K$.

We note that if, for $E$, we take $E_{2} \cong K$, then for a complex number $A$, we associate $A^{*}$ with the conjugate $\bar{A}$ of $A$.

LeMma 4.1. Let $f$ be an integrable function on $U$ into $B$, and $S$ and $W$ subspaces of $E$ such that for $x \in U, t \in S, f(x)(t) \in W$. Then for $x, y \in U$ such that $y-x \in S$, we have $[f(x)-f(y)](t)$ $\in W$ for all $t \in E$. 
Proof. For $x \in U$, set $g(x)=\int_{0}^{x} f(z) d z$. Let $x, y \in U$ such that $x \neq y$ and $y-x \in S$, and let $x=x_{0}<\cdots<x_{n+1}=y$, $n \in \omega$, be a subdivision of the interval $[x, y]$ of $E$. Then for $i=0$, $\cdots, n, \Delta x_{i}=x_{i+1}-x_{i} \in S$, and hence $f\left(x_{i}\right)\left(\Delta x_{i}\right) \in W$. Thus the Riemann sum $\sum_{0}^{n} f\left(x_{i}\right)\left(\Delta x_{i}\right) \in W$. Hence $\int_{x}^{y} f(z) d z \in W$ and $g(y)-g(x) \in W$.

Let $t \in E$ and $r \in R$ be such that $x+r t, y+r t \in U$. Then $(y+r t)-(x+r t)=y-x \in S$, and hence $g(y+r t)-g(x+r t)$ $\in W$. Thus for all $t \in E$,

$$
\begin{aligned}
f(y)(t)-f(x)(t)= & \lim _{r \rightarrow 0}[g(y+r t)-g(y)] r^{-1} \\
& -\lim _{r \rightarrow 0}[g(x+r t)-g(x)] r^{-1} \\
= & \lim _{r \rightarrow 0}\{[g(y+r t)-g(x+r t)]+[g(y)-g(x)]\} r^{-1} \\
\in W . &
\end{aligned}
$$

TheOREM 4.1. Let $H_{1}, \cdots, H_{n}, n \in \omega$, be invariant subspaces of $E$ distinct from $\{0\}$ such that $E=H_{1} \oplus \cdots \oplus H_{n}$ and let $f \in F$ be such that $S=\left(U \cap H_{1}\right) \oplus \cdots \oplus\left(U \cap H_{n}\right) \subseteq$ domain $f$. Then there exist integrable functions $f_{1}, \cdots, f_{n}$ such that for $i=1, \cdots, n$, $U \cap H_{i} \subseteq$ domain $f_{i} \subseteq H_{i}$ and range $f_{i} \subseteq T \mid H_{i}$, and such that for $x \in S, t \in E$,

$$
f(x)(t)=\sum_{i=1}^{n} f_{i}\left(x_{i}\right)\left(t_{i}\right),
$$

where $x=x_{1}+\cdots+x_{n}$ and $t=t_{1}+\cdots+t_{n}$ and $x_{i}, t_{i} \in H_{i}$ for $i=1, \cdots, n$.

Proof. For $i=1, \cdots, n$, set $g_{i}(x)(t)=f(x)\left(t_{i}\right)$ for $x \in S, t \in E$, and set $f_{i}=g_{i} \mid H_{i}$. Let $C$ be a closed path in $U$. Now for $i=1$, $\cdots, n, x \in S, t \in E$, we have $t_{i} \in H_{i}$ and hence $g_{i}(x)(t)=f(x)\left(t_{i}\right)$ $\in H_{i}$ and $\int_{C} g_{i}(z) d z \in H_{i}$. Thus $\left\{\int_{C} g_{i}(z) d z ; i=1, \cdots, n\right\}$ is a linearly independent set. Now for $x \in S, t \in E$,

$$
f(x)(t)=f(x)\left(\sum_{i=1}^{n} t_{i}\right) \sum_{i=1}^{n} f(x)\left(t_{i}\right)=\sum_{i=1}^{n} g_{i}(x)(t),
$$

and hence $0=\int_{C} f(z) d z=\int_{C}\left[\sum_{i=1}^{n} g_{i}(z)\right] d z=\sum_{i=1}^{n} \int_{C} g_{i}(z) d z$. Thus for $i=1, \cdots, n, \int_{C} g_{i}(z) d z=0$ and $g_{i}$ is integrable.

Let $i=1, \cdots, n$. Then for all $x \in S, t \in W_{i}=H_{1} \oplus \cdots \oplus$ $H_{i-1} \oplus H_{i+1} \oplus \cdots \oplus H_{n}$, we have $g_{i}(x)(t)=0$ and $x-x_{i} \in W_{i}$. Hence from Lemma 4.1, for all $x \in S, g_{i}(x)=g_{i}\left(x_{i}\right)$. Thus for $x \in S, t \in E$, 


$$
\begin{aligned}
f(x)(t) & =\sum_{k=1}^{n} g_{i}(x)(t)=\sum_{k=1}^{n} g_{i}(x)\left(t_{i}\right) \\
& =\sum_{k=1}^{n} g_{i}\left(x_{i}\right)\left(t_{i}\right)=\sum_{k=1}^{n} f_{i}\left(x_{i}\right)\left(t_{i}\right) .
\end{aligned}
$$

A subspace $H$ of $E$ is said to be an irreducible invariant subspace of $E$, if $H$ is an invariant subspace of $E$ distinct from $\{0\}$, and $H$ contains no proper invariant subspace distinct from $\{0\}$.

LEMMA 4.2. Suppose $H$ is an irreducible invariant subspace of $E$. Then $T_{0}=T \mid H$ is a field with the same dimension as $H$. Moreover a multiplication can be defined on $H$ such that $H$ becomes a field isomorphic to $T_{0}$, and such that for $A \in T_{0}$ there exists $a \in H$ such that $A(t)=$ at for all $t \in H$. Furthermore if the dimension of $H$ is two and $T$ is symmetric then $T_{0}$ and $H$ are isomorphic and isometric to $K$.

We observe that it follows from the fundamental theorem of algebra that $T_{0}$ is isomorphic (but not necessarily isometric) to $R$ or $K$, and thus that $H$ has dimension one or two. Suppose $H$ had dimension two. Let $f$ be an integrable function on an open set in $H$ into $T_{0}$. If we renorm $H$ and $T_{0}$ so that $H$ and $T_{0}$ are isometric to $K$, then $f$ becomes an integrable function from $K$ to $K$. Whence from Morera's theorem $f$ is analytic and can be expanded in power series. If $H$ has dimension one, then under suitable renorming $H$ and $T_{0}$ are isometric to $R$, and $f$ becomes an arbitrary continuous function from $R$ to $R$.

Proof of Lemma 4.2. Let $A \in T_{0}, A \neq 0$. Then $A$ is one-to-one on $H$. Indeed, for $D \in T$, setting $D_{0}=D \mid H$, we have $D[A(H)]=$ $D_{0}[A(H)]=A\left[D_{0}(H)\right] \subseteq A(H)$, and hence $A(H)$ is an invariant subspace of $H$; and consequently, since $H$ is irreducible, $A(H)=H$.

Let $x \in H \cap V$, and set $\theta(A)=A(x)$ for all $A \in T_{0}$. Now range $\theta$ $=\{A(x) ; A \in T\}$ is clearly an invariant subspace of $H$ and hence range $\theta=H$. Suppose for $A, B \in T_{0}, \theta(A)=\theta(B)$. Then $A(x)=$ $B(x)$ and $(A-B)(x)=0$. Thus $A-B$ is not one-to-one on $H$, and hence $A-B=0$, and thus $\theta$ is an isomorphism of $T_{0}$ onto $H$.

Let $A \in T_{0}, A \neq 0$. Since $A(H)=H$, there exists $y \in H$, such that $A(y)=x$. Now there exists $\alpha \in T_{0}$ such that $\theta(\alpha)=y$. Then $\alpha(x)=$ $y$ and $\boldsymbol{\theta}(A \boldsymbol{\alpha})=(A \boldsymbol{\alpha})(x)=A[\boldsymbol{\alpha}(x)]=A(y)=x$. Since $\theta(e)=x$, where $e$ is the identity element of $T_{0}$, we have $A \alpha=e$ and $\alpha=A^{-1}$, and thus $T_{0}$ is a field.

For $s, t \in H$, set $s t=\theta\left[\theta^{-1}(s) \theta^{-1}(t)\right]$. Let $A \in T_{0}$ and set $a=$ $A(x)$. Let $t \in H$ and set $B=\theta^{-1}(t)$. Then $B(x)=t$. Now $a t=$ $\theta\left[\theta^{-1}(a) \theta^{-1}(t)\right]=\theta[A B]=(A B)(x)=A[B(x)]=A(t)$. 
Now suppose $T$ is symmetric and $H$ had dimension two. Then $T_{0}$ is symmetric. Let $I$ be the element of $T_{0}$ such that $I^{2}=-e$. Now $\left(I^{*}\right)^{2}=\left(I^{2}\right)^{*}=(-e)^{*}=-e$ and thus $I^{*}= \pm I$. Now $I^{*}=-I$ since otherwise we would have $0<\|I x\|^{2}=[I x, I x]=\left[I I^{*} x, x\right]=$ $\left[I^{2} x, x\right]=-[x, x]=-1$. Let $r, s \in R$. Then for all $y \in V \cap H$, $\|(r+s I)(y)\|^{2}=\left[(r+s I)(r+s I)^{*} y, y\right]=[(r+s I)(r-s I) y, y]=$ $\left[\left(r^{2}+s^{2}\right) y, y\right]=\left(r^{2}+s^{2}\right)\|y\|^{2}=r^{2}+s^{2}$ and thus $\|r e+s I\|=$ $\left(r^{2}+s^{2}\right)^{1 / 2}$. Set $i=I(x)$. Then $[x, i]=[x, I x]=\left[I^{*} x, x\right]=$ $-[I x, x]=-[x, i]$ and thus $[x, i]=0$. Also $[i, i]=[I x, I x]=$ $\left[I I^{*} x, x\right]=\left[-I^{2} x, x\right]=[x, x]=1$. Thus $\|r x+s i\|^{2}=[r x+s i$, $r x+s i]=r^{2}[x, x]+2 r s[x, i]+s^{2}[i, i]=r^{2}+0+s^{2}$.

TheOREM 4.2. Suppose $T$ is symmetric and $f \in F$ with domain $U$. Then there exists a sequence $Z_{1}, \cdots, Z_{n}, n \in \omega$, in $\{R, K\}$ and $a$ sequence of functions $f_{1}, \cdots, f_{n}$ such that:

(1) For $i=1, \cdots, n$, if $Z_{i}=R$, then $f_{i}$ is an arbitrary continuous function on $(-1,1)$ into $R$.

(2) For $i=1, \cdots, n$, if $Z_{i}=K, f_{i}$ is a complex analytic function on $U \subseteq K$ into $K$.

(3) Setting $\phi(x)=\left[f_{1}\left(x_{1}\right), f_{2}\left(x_{2}\right), \cdots, f_{n}\left(x_{n}\right)\right]$ for $x=\left(x_{1}, \cdots, x_{n}\right)$ $\in W=Z_{1} \oplus \cdots \oplus Z_{n}$, there exists an isometry $\theta$ of $E$ onto $W$ and an isometry $\mu$ of $T$ onto $W$, such that $\mu^{-1}\{\phi[\theta(x)]\}=f(x)$ for all $x \in U$.

Proof. We decompose $E$. Since $E$ is finite dimensional, there exists an irreducible invariant subspace $E_{1}$ of $E$. Let $W_{1}$ be the orthogonal complement $\left\{y \in E ;(x, y)=0\right.$ for all $\left.x \in E_{1}\right\}$ of $E_{1}$. Then $E=E_{1} \oplus W_{1}$. Now for $y \in W_{1}, A \in T$, we have $A^{*} \in T$, and for all $x \in E_{1}, A^{*}(x) \in E_{1}$ and $[A(y), x]=\left[A^{*}(x), y\right]=0$; and consequently $A(y) \in W_{1}$. Thus $W_{1}$ is an invariant subspace of $E$. We next extract an irreducible invariant subspace $E_{2}$ of $E$ from $W_{1}$ and form the orthogonal complement $W_{2}$ of $E_{2}$ in $W_{1}$. Continuing in this manner until we exhaust $E$, we obtain a sequence $E_{1}, \cdots, E_{n}, n \in \omega$, of mutually orthogonal irreducible invariant subspaces of $E$ such that $E=E_{1} \oplus \cdots \oplus E_{n}$.

From Theorem 4.1, there exist integrable functions $g_{1}, \cdots, g_{n}$ such that for $i=1, \cdots, n$, domain $g_{i}=U \cap E_{i}$ and range $g_{i} \subseteq T \mid E_{i}$, and such that for $x \in U, t \in E, f(x)=\sum_{i=1}^{n} g_{i}\left(x_{i}\right)\left(t_{i}\right)$, where $x_{i}$ and $t_{i}$ are the projections of $x$ and $t$ respectively into $E_{i}$ for $i=1, \cdots, n$.

Let $i=1, \cdots, n$. Then from Lemma 4.2 , there is an isometry $\theta_{i}$ of $E_{i}$ onto an element $Z_{i}$ of $\{R, K\}$ and an isometry $\mu_{i}$ of $T_{i}=T \mid E_{i}$ onto $Z_{i}$, such that for $A \in T_{i}, x \in E_{i}$, we have $\left[\mu_{i}(A)\right] \cdot\left[\theta_{i}(x)\right]=$ $\theta_{i}[A(x)]$. For $x \in U \subseteq Z_{i}$, set $f_{i}(x)=\mu_{i}\left\{g_{i}\left[\theta_{i}{ }^{-1}(x)\right]\right\}$. Let $P$ be a 
path in $U \subseteq Z_{i}$ with endpoints $\alpha$ and $\beta$ and let $N: \alpha=x_{0}<\cdots<$ $x_{n+1}=\beta, n \in \omega$, be a subdivision of $P$, and let $R$ be the Riemann $\operatorname{sum} \sum_{0}^{n} f_{i}\left(x_{i}\right)\left(x_{i+1}-x_{i}\right)$. Then

$$
\begin{aligned}
\bar{R} & =\boldsymbol{\theta}_{i}^{-1}(\boldsymbol{R})=\sum_{0}^{n}\left\{\boldsymbol{\mu}_{i}^{-1}\left[f_{i}\left(x_{i}\right)\right]\right\}\left[\boldsymbol{\theta}_{i}^{-1}\left(x_{i+1}-x_{i}\right)\right] \\
& =\sum_{0}^{n}\left\{\boldsymbol{\mu}_{i}^{-1}\left[\boldsymbol{\mu}\left(g_{i}\left(\boldsymbol{\theta}_{i}^{-1} x_{i}\right)\right)\right]\right\}\left(y_{i+1}-y_{i}\right)=\sum_{0}^{n} g_{i}\left(y_{i}\right)\left(y_{i+1}-y_{i}\right)
\end{aligned}
$$

is a Riemann sum for the subdivision $\bar{N}: \bar{\alpha}=y_{0}<\cdots<y_{n+1}=$ $\bar{\beta}$ of the path $\bar{P}=\theta_{i}^{-1}(P)$ of $E_{i}$, where $y_{i}=\theta_{i}^{-1}\left(x_{i}\right)$ for $i=0,1, \cdots$, $n+1$. Now norm $N=$ norm $\bar{N}$. Hence taking the limit as norm $N$ converges to zero we obtain $\theta_{i}-1\left[\int_{\alpha_{p}}^{\beta} f(x) d x\right]=\int_{\bar{\alpha}_{\bar{p}}}^{\bar{\beta}} g(y) d y$. Thus $f_{i}$ is an integrable function from $Z_{i}$ into $Z_{i}^{p}$.

We observe that if $\theta_{i}$ and $\mu_{i}$ are merely required to be isomorphisms rather than isometries then there exists a number $C=\left\|\theta_{i}{ }^{-1}\right\|<\infty$ such that norm $\bar{N} \leqq C \cdot($ norm $N)$. Hence as norm $N \rightarrow 0$, norm $\bar{N} \rightarrow 0$, and the desired equality of integrals exists.

For $z \in W$, set $\phi(x)=\left[f_{1}\left(x_{1}\right), \cdots, f_{n}\left(x_{n}\right)\right]$. For $x \in E$, set $\theta(x)=$ [ $\left.\theta_{1}\left(x_{1}\right), \cdots, \theta_{n}\left(x_{n}\right)\right]$ and for $A \in T$, writing $A_{i}$ for $A \mid E_{i}$ for $i=1$, $\cdots, n$, set $\mu(A)=\left[\mu_{1}\left(A_{1}\right), \cdots, \mu_{n}\left(A_{n}\right)\right]$. Then $\mu^{-1}\{\phi[\theta(x)]\}=$ $f(x)$ for all $x \in U$.

5. Analyticity. The principal result of this section, Theorem 5.3, is that if $T$ is presumed to be semisimple then a necessary and sufficient condition for analyticity is that no element of $T$ is of rank one. It is conjectured that the requirement of semisimplicity can be dispensed with. Indeed regardless of whether or not $T$ is semisimple we have from Theorem 5.1 that if $T$ contains an element of rank one, then $F$ contains a nondifferentiable element.

We observe that a necessary and sufficient condition for $T$ to be semisimple is that $T$ contain no nilpotent elements, i.e. elements $x$, such that $x \neq 0$, but $x^{k}=0$ for some $k \in \omega$.

A definition of analyticity suitable for the real variable context of this paper has been given by this author [2], [5], [6]. If the elements of $F$ satisfy a uniform Lipschitz condition descended from Schwarz's lemma in complex function theory, then the elements of $F$ are (Fréchet) differentiable, indeed infinitely (Fréchet) differentiable, and expandable in power series.

Let $Z$ be a family of continuous functions on open subsets of $E$ into a Banach space $C$. $Z$ is called a TR family if:

(1) $f+g \in Z$ for $f, g \in Z$. 
(2) $r f \in Z$ for $f \in Z, r \in R$.

(3) For $f \in Z$, $S$ an open set in $E$, setting $g=f \mid S$, $g \in Z$.

(4) For $f \in Z, y \in E$, the translate $f_{y}$ of $f$ lies in $Z$, where $f_{y}(x)=$ $f(x-y)$ for $x \in y+($ domain $f)$.

(5) For $f \in Z, r>0$, setting $g(x)=f(r x)$ for $x \in r^{-1}($ domain $f)$, we have $g \in Z$.

Trivially $F$ is a TR family.

$Z$ is called a TRL family if $Z$ is a TR family and

(6) there exists $N>0$, such that for $f \in Z, \bar{U} \subseteq$ domain $f, x \in$ domain $f$, we have

$$
\|f(x)-f(0)\| \leqq N \sup \{\|f(t)\| ; t \in U\}\|x\| .
$$

We denote the least upper bound of the family of all such numbers $N$ by $N(Z)$.

If $Z$ is a TRL family, $f \in Z$, and $z_{0} \in$ domain $f$, then for some $\delta>0$, the series $\sum_{0}^{\infty} f_{z_{0}}^{(n)}\left(z-z_{0}\right) / n$ ! converges uniformly on $U_{z_{0}}(\delta)$ to $f(z)$, where $f_{z_{0}}^{(n)}$ is a homogeneous function on $E$ into $T$ of degree $n$, derived from the $n$th Fréchet derivative of $f$ at $z_{0}$, for $n \in \omega$.

THEOREM 5.1. A necessary condition for $F$ to be a TRL family of analytic functions is that no element of $T$ have a one-dimensional range.

Proof. Let $A \in T$, such that $A(E)$ is one dimensional, and set $H=\{x \in E ; A(x)=0\}$. Then the dimension of $H$ is $p-1$. Let $w$ be an element of $E$ such that $w_{0}=A(w) \neq 0$. Then $E=\{r w ; r \in R\}$ $\oplus H$. Let $h$ be an arbitrary continuous function with domain $R$ into $R$. For $r \in R$, set $u(r)=\int_{0}^{r} h(t) d t$. For $z=r w+x, r \in R, x \in H$, set $f(z)=h(r) A$ and set $\phi(z)=u(r) w_{0}$. Then for $z=r w+x$, $\rho=s w+y, r, s \in R, \quad x, y \in H$, we have $A(\rho)=A(s w)+A(y)=$ $s w_{0}+0$, and $\phi_{z}{ }^{\prime}(\boldsymbol{\rho})=\left[u^{\prime}(r) s\right] w_{0}=[h(r)] s w_{0}=h(r) A(\rho)=$ $[f(z)](\rho)$ and $\phi^{\prime}(z)=f(z)$. Then as in the examples of $\$ 3$ for any path $P \subseteq E$ with endpoints $\alpha$ and $\beta, \int_{\alpha_{p}}^{\beta} f(z) d z=\phi(\beta)-\phi(\alpha)$, and thus for any closed path $C \subseteq E, \int_{C} f(z) d z=0$, and $f$ is integrable. Clearly since $h$ need not satisfy any Lipschitz conditions, $f$ need not satisfy condition (6) of the definition of a TRL family for any $N>0$.

Let $G$ be the family of all functions $g$ on open sets $S \subseteq E$ into $E$, such that there exists $f \in F$ with domain $S$, such that if $x$ and $y$ are points of the same component of $S$, then $g(y)-g(x)=\int_{x}^{y} f(z) d z$.

For $f \in F, H$ a subspace of $E$, set $f_{H}(z)=f(z)|H \in T| H$, and set $\left.f\right|^{\prime} H=f_{H} \mid H$. Set $\left.F\right|^{\prime} H=\left\{\left.f\right|^{\prime} H ; f \in F\right\}$.

TheOREM 5.2. Let $H$ be a subspace of $E$, such that $\left.F\right|^{\prime} H$ and $G \mid H$ are TRL families of analytic functions, and such that for $A \in T, A(x)$ 
$=0$ for all $x \in H$ implies $A(x)=0$ for all $x \in E$. Then $F$ and $G$ are TRL families of analytic functions.

Proof. For $A \in T$, set $\theta(A)=A \mid H$, and set $T_{0}=T \mid H$. If for $A, B \in T, \theta(A)=\theta(B)$, then for $x \in H, A(x)=B(x)$ and $(A-B)(x)$ $=0$, and hence by hypothesis, $A-B=0$ and $A \equiv B$. Thus $\theta$ is an isomorphism and there exists $N_{1}>0$ such that for $A \in T_{0},\left\|\theta^{-1}(A)\right\|$ $\leqq N_{1}\|A\|$.

Clearly $G$ is a TR family. We now show that $G$ is a TRL family. Let $f \in F, g \in G$, such that $\bar{U} \subseteq$ domain $f=$ domain $g$, and $g(y)$ $-g(x)=\int_{x}^{y} f(z) d z$ for $x, y \in U$. Let $x_{0} \in U$ and set $r_{0}=1-\left\|x_{0}\right\|$. Then $r_{0} U+x_{0} \subseteq U$. For $x \in U$, set $h(x)=g\left(r_{0} x+x_{0}\right)$. Then $h \in G$. Set $M=\{\|g(t)\| ; t \in U\}$, set $G_{0}=G \mid H$, and set $N_{0}=N\left(G_{0}\right)$. Then for $x \in U \cap H$,

$$
\|h(x)-h(0)\| \leqq N_{0} \sup \{\|h(t)\| ; t \in U \cap H\}\|x\| \leqq N_{0} M\|x\| .
$$

Now for $\alpha \in V \cap H, 0<r<r_{0}$,

$$
\begin{aligned}
f\left(x_{0}\right)(\boldsymbol{\alpha}) & =\lim _{s \rightarrow 0}\left[g\left(x_{0}+s \boldsymbol{\alpha}\right)-g\left(x_{0}\right)\right] s^{-1} \\
& =\lim _{s \rightarrow 0}\left[h\left(s r_{0}^{-1} \boldsymbol{\alpha}\right)-h(0)\right] s^{-1},
\end{aligned}
$$

and

$$
\left\|\left[h\left(s r_{0}{ }^{-1} \boldsymbol{\alpha}\right)-h(0)\right] s^{-1}\right\| \leqq M N_{0} s^{-1}\left\|s r_{0}{ }^{-1} \alpha\right\|=M N_{0} r_{0}{ }^{-1}
$$

and thus $\left\|f\left(x_{0}\right)(\alpha)\right\| \leqq M N_{0} r_{0}{ }^{-1}$, and hence $\left\|\theta f\left(x_{0}\right)\right\| \leqq M N_{0} r_{0}{ }^{-1}$ and $\left\|f\left(x_{0}\right)\right\| \leqq M N_{0} N_{1} r_{0}^{-1}$. Then for $x \in U\left(\frac{1}{2}\right)$,

$$
\begin{aligned}
\|g(x)-g(0)\| & =\left\|\int_{0}^{x} f(z) d z\right\| \leqq \int_{0}^{x}\|f(z)\| d s \\
& \leqq \int_{0}^{x} M N_{0} N_{1}\left(\frac{1}{2}\right)^{-1} d s=2 M N_{0} N_{1}\|x\|
\end{aligned}
$$

and thus for $x \in U$,

$$
\|g(x)-g(0)\| \leqq N M\|x\|,
$$

where $N=\max \left\{2 N_{0} N_{1}, 4\right\}$. Thus $G$ is a TRL family and hence by [2], $F$ is a TRL family.

LeMMA 5.1. Let $f$ be an integrable function on $U$ such that for $x \in U, f(x)=r(x)$ e, where $r$ is a continuous function on $U$ into $R$. Then $f$ is constant.

Proof. Let $x, y \in U, x \neq y$ and set $S=W=\{s(y-x) ; s \in R\}$. 
Then for $z \in U, t \in S, f(z)(t)=r(z) t \in W$, and hence from Lemma 5.2, $[f(y)-f(x)](t) \in S$ for all $t \in E$. Thus $[r(y)-r(x)] t$ is a multiple of $y-x$ for all $t \in E$, and hence $r(y)-r(x)=0$ and $r(y)=r(x)$.

THEOREM 5.3. If $T$ is semisimple, then a necessary and sufficient condition that $F$ be a TRL family of analytic functions is that no element of $T$ have rank one.

Proof. Necessity follows from Theorem 5.1. Let $\mathcal{A}_{0}=\{A(E)$; $A \in T, A \neq 0\}$ and let $\&$ be the family of all $H \in \mathcal{H}_{0}$ such that $\sigma \in \mathcal{H}_{0}, \sigma \subseteq H$, implies $\sigma=H$. Clearly $\mathcal{H}$ is nonempty. Let $W$ be the subspace of $E$ generated by the union of the elements of $d$. To show sufficiency we shall first show that $\left.F\right|^{\prime} W$ and $G \mid W$ are TRL families of analytic functions. We then exploit the semisimplicity of $T$ to show that the elements of $T$ are determined by their behavior on $W$ allowing us to employ Theorem 5.2.

Let $H \in \mathcal{A}$ and set $T_{0}=T \mid H$. Suppose $T_{0}$ is one dimensional. Then all elements of $T_{0}$ are of the form $r e_{0}$, where $r \in R$, and $e_{0}=$ $e \mid H$. Since $H$ is not one dimensional, from Lemma 5.1, the elements of $\left.F\right|^{\prime} H$ are locally constant functions and thus trivially $\left.F\right|^{\prime} H$ and $G \mid H$ are TRL families.

Suppose $T_{0}$ is not one dimensional. Employing Theorem 4.2, we shall show that any element $\left.f \in F\right|^{\prime} H$ can be expressed locally as a direct sum of complex analytic functions from $K$ into $K$, thus yielding that $\left.F\right|^{\prime} H$ and $G \mid H$ are TRL families.

Let $x \in H_{0}=H-\{0\}$. Then $\bar{x}=\{P(x) ; P \in T\}$ is an invariant subspace of $H$. Let $\boldsymbol{\sigma}$ be an irreducible invariant subspace of $\bar{x}$. For $P \in T_{0}=T \mid H$, set $\theta(P)=P \mid \sigma$. Suppose for some $P \in T$, $P_{0}=P \mid H$, that $\theta\left(P_{0}\right)=0$. Then $P(\sigma)=\{0\}$ and $P$ is not one-to-one on $H$. Then $H \neq P(H) \subseteq H$, and $H=P(A)$ and $P(H)=P[A(E)]$ $=(P A)(E)=$ range $P A$ for some $A \in T$. From the minimality of $H, P(H)=\{0\}$, and $P_{0}=0$ and thus $\theta$ is an isomorphism. Thus $T_{1}=T_{0}|\sigma=T| \sigma$ is isomorphic to $T_{0}$ and hence $T_{1}$ does not have dimension one. From Lemma 4.2, $T_{1}$, and hence $T_{0}$ is a field isomorphic to $K$. Let $y \in \sigma, y \neq 0, z \in \bar{x}$. Then there exist $P, Q \in T_{0}$ such that $y=P(x)$ and $z=Q(x)$. Whence $z=\left(Q P^{-1}\right)(y)$. Then $\bar{x} \subseteq \sigma$ and $\bar{x}=\sigma$ and $\bar{x}$ is irreducible. Clearly $T \mid \bar{x}$ is isomorphic to $K$. Let $\left.f \in F\right|^{\prime} H$.

Now $\left\{\bar{x} ; x \in H_{0}\right\}$ generates $H$. Hence there exist $x_{1}, \cdots, x_{k} \in H_{0}$, $k \in \omega$, such that $H=\bar{x}_{1} \oplus \cdots \oplus \bar{x}_{k}$. Then following the proof of Theorem 4.2, there exists an isomorphism $\pi$ of $H$ onto a complex Hilbert space $H^{\prime}$ of complex dimension $k$, and an isomorphism $\mu$ of 
$T_{0}$ onto $K$, such that $g=\mu f \pi^{-1}$ is a complex analytic function on $\pi($ domain $f)$ into $K$.

There exist $\rho, \delta>0$, such that $\pi[U(\rho) \cap H] \subseteq U(\delta) \subseteq \pi(U \cap H)$. Then $\pi[U(\boldsymbol{\rho}) \cap H] \subseteq$ domain $g$. Let $x \in U(\boldsymbol{\rho}) \cap H, x^{\prime}=\pi(x)$. Then from complex function theory,

$$
\begin{aligned}
\| f(x) & -f(0)\|\leqq\| \mu^{-1}\|\cdot\| g\left(x^{\prime}\right)-g(0) \| \\
& \leqq 2\left\|\mu^{-1}\right\| \sup \{\|g(t)\| ; t \in U(\delta)\} \delta^{-1}\left\|x^{\prime}\right\| \\
& \leqq 2\left\|\mu^{-1}\right\|\left[\|\mu\| \sup \left\{\|f(t)\| ; t \in \pi^{-1}[U(\delta)]\right\}\right] \delta^{-1}[\|\tau\| \cdot\|x\|] \\
& \leqq N M\|x\|,
\end{aligned}
$$

where $M=\sup \{\|f(t)\| ; t \in U \cap H\}$ and

$$
N=\max \left\{2\left\|\mu^{-1}\right\| \cdot\|\mu\| \cdot\|\pi\| \delta^{-1}, 2 / \rho\right\} .
$$

For $x \in U(\rho) \cap H,\|x\| \geqq \rho$, we have

$$
\|f(x)-f(0)\| \leqq 2 M=[2 M / \rho] \rho \leqq[2 M / \rho]\|x\| \leqq N M\|x\| .
$$

Thus $F_{H}=\left.F\right|^{\prime} H$ is a TRL family and $N\left(F_{H}\right) \leqq N$.

$W$ can be expressed as the direct sum of a finite subcollection $\left\{H_{1}, \cdots, H_{n}\right\}$ of $\mathcal{H}, n \in \omega$. Then for $x \in W$, there exist unique elements $x_{1}, \cdots, x_{n}$ of $W$, such that $x_{i} \in H_{i}$ for $i=1, \cdots, n$, and $x=x_{1}+\cdots+x_{n}$. Moreover there exists $\rho>1$ such that for $x \in W, i=1, \cdots, n$, we have $\left\|x_{i}\right\| \leqq \rho\|x\|$. For $i=1, \cdots, n$, set $N_{i}=N\left[\left.F\right|^{\prime} H_{i}\right]$. Let $\left.f \in F\right|^{\prime} W, \bar{U} \cap W \subseteq \operatorname{domain} f$.

For $i=1, \cdots, n$, set $f_{i}=\left.f\right|^{\prime} H_{i}$. Then from Theorem 4.1, for $z \in U\left(\rho^{-1}\right) \cap W, \quad t \in W, \quad f(z)(t)=\sum_{i=1}^{n} f_{i}\left(z_{i}\right)\left(t_{i}\right)$. Whence for $x \in U\left(\rho^{-1}\right) \cap W$, we have $x_{1}, \cdots, x_{n} \in U$ and

$$
\begin{aligned}
\|f(x)-f(0)\| & \leqq \sum_{i=1}^{n}\left\|f_{i}\left(x_{i}\right)-f_{i}(0)\right\| \\
& \leqq \sum_{i=1}^{n} N_{i} \sup \left\{\left\|f_{i}(s)\right\| ; s \in U \cap H_{i}\right\}\left\|x_{i}\right\| \\
& \leqq \sum_{i=1}^{n} N_{i} M[\rho\|x\|] \leqq N M\|x\|,
\end{aligned}
$$

where $N=\max \left\{\rho \sum_{i=1}^{n} N_{i}, 2 \rho\right\}$ and

$$
M=\sup \{\|f(s)\| ; s \in U \cap W\} .
$$

Trivially (1) holds for $x \in U \cap W,\|x\| \geqq \rho^{-1}$, and thus $\left.F\right|^{\prime} W$ is a 
TRL family. By a similar argument we show that $G \mid W$ is a TRL family.

Let $A$ be an arbitrary element of $T$ such that $A(x)=0$ for all $x \in W$. If we show that $A \equiv 0$, then the theorem follows from Theorem 5.2. Suppose $A \neq 0$ and consider the sequence $E \supseteq A(E)$ $\supseteq A^{2}(E) \supseteq \cdots \quad$ Since $T$ is semisimple, there exists $k \in \omega$ such that $A^{k}(E)=A^{k+1}(E)=A\left[A^{k}(E)\right] \neq\{0\}$ and $A$ is one-to-one on $A^{k}(E)$. Now there exists $H \in \mathcal{A}$ such that $H \subseteq A^{k}(E)$. Whence $A(H) \neq\{0\}$. But $H \subseteq W$ and $A(H) \subseteq A(W)=\{0\}$.

REMark. The method of proof of Theorem 5.3 suggests that the requirement

(1) No element of $T$ has rank one;

be replaced by

(2) $\left.F\right|^{\prime} H$ is a TRL family for all one-dimensional elements $H$ of $\not_{0}$.

We observe from [6] that if (2) holds, there exists $n \in \omega$, such that $\left.F\right|^{\prime} H$ is isomorphic to the family of polynomials and of restrictions of polynomials to open subsets of $R$ into $R$ of degree less than $n$.

It can be readily shown that the requirement of semisimplicity combined with (2) yields the conclusion that (1) must hold and thus that $F$ is a TRL family. Indeed suppose there exists $A \in T$, such that $H=A(E)$ has dimension one. Now if $A(H)=\{0\}$, then $A^{2}(E)=A(H)$ $=\{0\}$, and hence, since $T$ is presumed semisimple, $A=0$. Thus $A(H)=$ $H$. Let $w \in H, w \neq 0$, let $h$ be a continuous nondifferentiable function on $R$ into $R$, and for $x \in E$, set $f(x)=h\left(x^{\prime}\right) A$, where $x^{\prime} \in R$, $x^{\prime} w=A(x)$. Then as in the proof of Theorem 5.1, $f$ is integrable and nondifferentiable, contradicting (2).

Neither semisimplicity nor condition (2) is sufficient separately to give the conclusion that $F$ is a TRL family as the following examples show. For $E$ we take $R \oplus R$. For $(x, y) \in E$, set $P_{1}(x, y)=(x, 0)$ and $P_{2}(x, y)=(y, 0)$. For $i=1,2$, set $T_{i}=\left\{r e+s P_{i} ; r, s \in R\right\}$, and let $F_{i}$ be the family of integrable functions on open subsets of $E$ into $T_{i}$. Both $P_{1}$ and $P_{2}$ have one-dimensional ranges, and hence from Theorem 5.1, $F_{1}$ and $F_{2}$ are not TRL families of analytic functions. We show that $T_{1}$ is semisimple and that $\left.F_{2}\right|^{\prime} H$ is a family of constant functions and hence trivially a TRL family, where $H=\{(x, 0) ; x \in R\}$ is, as we shall show, the only one-dimensional element of $\left\{A(E) ; A \in T_{2}\right\}$.

Let $r, s \in R, k \in \omega, k>1$, and set $A=r e+s P_{1}$. Suppose $A^{k}=0$. Then for some $c \in R, \quad 0=A^{k}=\left(r e+s P_{1}\right)^{k}=r^{k} e+\left(r c+s^{k}\right) P_{1}$ and hence $r^{k}=0$ and $r c+s^{k}=0$. Then $r=s=0$ and $A=0$, and $T_{1}$ is semisimple.

Let $A^{\prime} \in T_{2}$ and set $H^{\prime}=A^{\prime}(E)$. Suppose $H^{\prime} \neq H$ and $H^{\prime}$ is one dimensional. Then $P_{2}\left(H^{\prime}\right) \neq\{0\}$ and $H \cap H^{\prime}=\{0\}$. Since $H^{\prime}$ is 
an invariant subspace of $E, P_{2}\left(H^{\prime}\right) \subseteq H^{\prime}$. Now $P_{2}\left(H^{\prime}\right) \subseteq P_{2}(E)=H$ and thus $P_{2}\left(H^{\prime}\right) \subseteq H \cap H^{\prime}=\{0\}$. But then $P_{2}\left(H^{\prime}\right)=\{0\}$. Thus $A^{\prime}(E)=H$.

Let $\alpha$ and $\beta$ be continuous functions on $U$ into $R$ such that $\alpha e+$ $\beta P_{2}$ is integrable. Now $f=\alpha P_{2}=\left(\alpha e+\beta P_{2}\right) P_{2}$ is integrable and $f(z)(H)=\{0\}$ for $z \in U$, and hence applying Lemma 4.1, $f=\alpha P_{2}$ is constant on $H$. Now for $z_{1}, z_{2} \in H$,

$$
\alpha\left(z_{1}\right) P_{2}(0,1)=\alpha\left(z_{2}\right) P_{2}(0,1) \text { and } \alpha\left(z_{1}\right)(1,0)=\alpha\left(z_{2}\right)(1,0) \text {, }
$$

and thus $\alpha\left(z_{1}\right)=\alpha\left(z_{2}\right)$ and $\left.\alpha e\right|^{\prime} H$ is constant. Trivially $\left.\beta P_{2}\right|^{\prime} H$ is a null operator valued constant function. Thus $\left.F_{2}\right|^{\prime} H$ is a TRL family.

It is conjectured that the requirement that there exist no onedimensional range spaces is a necessary and sufficient condition of analyticity. If this conjecture fails we propose the condition that there exist no one-dimensional invariant subspaces. This stronger condition forces $E$ and all invariant subspaces of $E$ to be of even dimension, causing one to suspect that some sort of complexification of $E$ and $T$ is possible.

\section{REFERENCES}

1. L. Heffter, Begründung der Funktionentheorie auf alten und neuen Wegen, Springer-Verlag, Berlin, 1955. MR 16, 807.

2. K. O. Leland, A characterization of analyticity, Duke Math. J. 33 (1966), 551-565. MR 33 \#5914.

3. - A polynomial approach to topological analysis, Compositio Math. 17 (1967), 291-298. MR 34 \#4515.

4. - Topological analysis of differentiable transformations, Compositio Math. 18 (1967), 189-200. MR 36 \#5364.

5. — A characterization of analyticity. II, Proc. Amer. Math. Soc. 19 (1968), 519-527. MR 38 \#3395.

6. - A characterization of analyticity. III, J. Math. Mech. 18 (1968/69), 109-123. MR 38 \#3396.

7. _ Algebras of integrable functions, Preprint (submitted).

8. _ Maximum modulus theorems for algebras of operator valued functions, Pacific J. Math. 39 (1971).

9. A. J. Macintyre and W. John Wilbur, A proof of the power series expansion without differentiation theory, Proc. Amer. Math. Soc. 18 (1967), 419-424. MR 35 \#5638.

10. M. A. Naimark, Normed rings, GITTL, Moscow, 1956; English transl., Noordhoff, Groningen, 1959. MR 19, 870; MR 22 \#1824.

Illinois Institute of Technology, Chicago, Illinois 60616 